\title{
ARTICLE
}

\section{TSPO ligands prevent the proliferation of vascular smooth muscle cells and attenuate neointima formation through AMPK activation}

\author{
Lian-pan $\mathrm{Wu}^{1,2}$, Zheng-fan Gong ${ }^{1,2}$, He Wang ${ }^{1,2}$, Zhong-shu Zhou ${ }^{1,2}$, Ming-ming Zhang ${ }^{1,2}$, Chao Liu ${ }^{1,2}$, Hong-mei Ren ${ }^{1,2}$, Jian Yang ${ }^{1,2}$, \\ Yu $\operatorname{Han}^{1,2}$ and Chun-yu Zeng ${ }^{1,2}$
}

\begin{abstract}
Abnormal growth of the intimal layer of blood vessels (neointima formation) contributes to the progression of atherosclerosis and in-stent restenosis. Recent evidence shows that the $18-\mathrm{kDa}$ translocator protein (TSPO), a mitochondrial membrane protein, is involved in diverse cardiovascular diseases. In this study we investigated the role of endogenous TSPO in neointima formation after angioplasty in vitro and in vivo. We established a vascular injury model in vitro by using platelet-derived growth factor-BB (PDGF$\mathrm{BB}$ ) to stimulate rat thoracic aortic smooth muscle cells (A10 cells). We found that treatment with PDGF-BB (1-20 ng/mL) dosedependently increased TSPO expression in A10 cells, which was blocked in the presence of PKC inhibitor or MAPK inhibitor. Overexpression of TSPO significantly promoted the proliferation and migration in A10 cells, whereas downregulation of TSPO expression by siRNA or treatment with TSPO ligands PK11195 or Ro5-4864 (10 4 nM) produced the opposite effects. Furthermore, we found that PK11195 (10-104 nM) dose-dependently activated AMPK in A10 cells. PK11195-induced inhibition on the proliferation and migration of PDGF-BB-treated A10 cells were abolished by compound C (an AMPK-specific inhibitor, $10^{3} \mathrm{nM}$ ). In rats with balloon-injured carotid arteries, TSPO expression was markedly upregulated in the carotid arteries. Administration of PK11195 ( $3 \mathrm{mg} / \mathrm{kg}$ every 3 days, ip), starting from the initial balloon injury and lasting for 2 weeks, greatly attenuated carotid neointima formation by suppressing balloon injury-induced phenotype switching of VSMCs (increased a-SMA expression). These results suggest that TSPO is a vascular injury-response molecule that promotes VSMC proliferation and migration and is responsible for the neointima formation after vascular injury, which provides a novel therapeutic target for various cardiovascular diseases including atherosclerosis and restenosis.
\end{abstract}

Keywords: neointima formation; vascular smooth muscle cells; proliferation; TSPO; AMPK; PDGF-BB; PK11195; Ro5-4864; compound C; balloon-injured carotid arteries

Acta Pharmacologica Sinica (2020) 41:34-46; https://doi.org/10.1038/s41401-019-0293-x

\section{INTRODUCTION}

The abnormal growth of the intimal layer of blood vessels (neointima formation) is a prevalent and severe pathophysiological process that contributes to the progression of atherosclerosis and in-stent restenosis [1]. Vascular smooth muscle cells (VSMCs) are the main constituents of the neointima in restenotic and atherosclerotic lesions. The molecular mechanisms underlying this complex series of changes are not completely understood. It is generally accepted that VSMCs undergo phenotype transformation, proliferate, migrate and secrete extracellular matrix components, resulting in neointima formation in response to injury or other stimuli $[2,3]$. During neointima formation, growth factors, such as transforming growth factor- $\beta$ and platelet-derived growth factor-BB (PDGF-BB), are produced locally [4]. Currently, treatments aimed at suppressing neointima formation are extremely limited. Thus, elucidating the mechanisms governing VSMC growth modulation is necessary for understanding the pathogenesis and progression of many proliferative vascular diseases and ultimately identifying novel therapeutic targets to treat these diseases.

The translocator protein (TSPO) is an $18 \mathrm{kDa}$ protein distributed in the outer mitochondrial membrane. TSPO is highly conserved in sequence and function among diverse organisms $[5,6]$. TSPO expression is altered readily in various cells exposed to physicochemical stimuli or pro-pathological factors. The roles of TSPO in apoptosis [7], inflammation [8], HIV biosynthesis [9], cancer [10], and Alzheimer's disease [11] have been investigated. In particular, TSPO is involved in several cardiovascular diseases, such as ischemia-reperfusion injury, cardiac arrhythmia, cardiac hypertrophy, and atherosclerosis [4, 12, 13]. PK11195 and Ro54864 are two well-studied TSPO ligands [14]. In vitro studies have shown that PK11195 binds to macrophage-rich human atherosclerotic plaques [15]. Radioactively labeled PK11195 has been used to image atherosclerosis $[16,17]$ and has indicated that TSPO

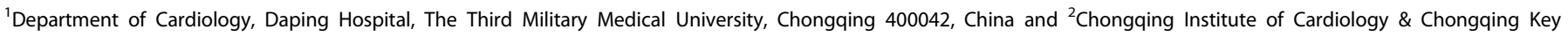
Laboratory of Hypertension Research, Chongqing 400042, China

Correspondence: Yu Han (hanc2-823@126.com) or Chun-yu Zeng (chunyuzeng01@163.com)

Co-first author: Lian-pan Wu, Zheng-fan Gong

Received: 7 March 2019 Accepted: 17 July 2019

Published online: 12 September 2019 
is upregulated and activated in the plaque region, whereas the role of TSPO is still elusive. Considering the role of TSPO in the regulation of tumor cell proliferation and the abundance of TSPO in the inflammatory regions of atherosclerotic plaques, we infer that there may be a link between TSPO and the aberrant proliferation of VSMCs after angioplasty.

In this study, we investigated the potential role of endogenous TSPO in the proliferation and migration of A10 cells treated with PDGF-BB. Then, we used the TSPO ligands PK11195 and Ro5-4864 to investigate their effects on PDGF-BB-mediated proliferation in A10 cells and explored the underlying mechanism. Furthermore, we tested the effect of PK11195 on neointimal hyperplasia induced by balloon injury in Sprague-Dawley (SD) rats.

\section{MATERIALS AND METHODS}

Rat carotid artery balloon injury model

Male SD rats $(250 \pm 50 \mathrm{~g}$ body weight) were purchased from the Center of Experimental Animals of Third Military Medical University (Chongqing, China). All procedures were approved by the Animal Use Subcommittee at the Third Military Medical University. SD rats were anesthetized using a mixture of ketamine $(80 \mathrm{mg} / \mathrm{kg})$ and xylazine $(12 \mathrm{mg} / \mathrm{kg})$, and then the left common and external carotid arteries were exposed and isolated. A $2 \mathrm{~F}$ Fogarty catheter (Edwards Life Sciences, Irvine, CA, USA) was introduced into the common carotid artery through an arteriotomy in the external carotid artery and inflated to 1.0-1.5 atm. A $10-\mathrm{mm}$ injury was induced by withdrawing the catheter four times. The external carotid artery was then untied, and blood flow was restored. After the balloon injury, SD rats were intraperitoneally injected with PK11195 (3 mg/kg every 3 days) or a similar volume of saline $(2 \mathrm{~mL} / \mathrm{kg})$ for 2 weeks.

\section{Materials and agents}

A TSPO antibody was purchased from Thermo Fisher Scientific (PA5-75544, Shanghai, China). Antibodies against total AMPKa (\#5232), phosphorylated AMPKa-172 (\#50081), a-SMA (\#19245), cyclin D1 (\#2922), cyclin E (\#4129), PCNA (\#2586), H3 (\#4499) and GAPDH (\#5174) were obtained from Cell Signaling Technology (Beverly, MA, USA). Infrared-labeled donkey anti-rabbit IRDye 800 and goat anti-mouse IRDye 800 were purchased from Li-Cor Biosciences (Beijing, China). Secondary antibodies conjugated to Cy3 and FITC were purchased from Jackson Immuno-Research (West Grove, PA, USA). Cell culture dishes were obtained from NEST Biotechnology Co., Ltd (Shanghai, China). Different antagonists were used in this study, including protein kinase $C$ (PKC) inhibitor peptide 19-31 (catalog no.: 05-23-4904, Calbiochem Co., Darmstadt, Germany), protein kinase A (PKA) inhibitor 14-22 amide (catalog no.: 476485, Calbiochem Co., La Jolla, CA, USA), the dihydropyridine calcium channel blocker nicardipine (catalog no.: N7510, Sigma Co., St. Louis, MO, USA), the mitogen-activated protein (MAP) kinase inhibitor PD98059 (catalog no.: P215, Sigma Co., St. Louis, MO, USA), and the PKC activator phorbol 12myristate 13-acetate (PMA) (catalog no.: P8139, Sigma Co., St. Louis, MO, USA).

\section{Histopathological analysis}

Hematoxylin-eosin (HE) staining was performed as previously described [18]. Briefly, the animals were anesthetized as described above, the carotid arteries were fixed and removed, and six cross sections were cut from approximately the middle of each artery. The sections were stained with HE to calculate the neointimal area and the ratio of intima-to-media area.

\section{Immunofluorescence staining}

Carotid artery sections and coverslipped A10 cells were prepared for immunofluorescence staining using a protocol described previously [18]. The samples were permeabilized with $0.2 \%$ Triton
$\mathrm{X}-100$ in phosphate-buffered saline (PBS) for $10 \mathrm{~min}$. Endogenous peroxidase activity was quenched, and the sections were blocked in $10 \%$ horse serum in PBS for $1 \mathrm{~h}$. Primary antibodies (TSPO, aSMA, PCNA or Mito-Tracker, 1:100) were applied overnight at $4{ }^{\circ} \mathrm{C}$, and a secondary antibody was applied for $1 \mathrm{~h}$ at room temperature. Samples that were incubated with an isotypematched control antibody were used as negative controls. Images were taken with a confocal laser scanning microscope, and the data were analyzed.

\section{Cell culture}

A rat thoracic aortic smooth muscle cell line (A10 cells) was obtained from American Type Culture Collection. A10 cells were cultured in Dulbecco's modified Eagle's medium (DMEM) supplemented with $10 \%$ fetal bovine serum (FBS) at $37^{\circ} \mathrm{C}$ in a humidified $5 \% \mathrm{CO}_{2}$ atmosphere. Cultured cells were grown to $60 \%-70 \%$ confluence before being starved in FBS-free DMEM for $24 \mathrm{~h}$. The starved cells were cultured in DMEM containing 10\% FBS with various concentrations $\left(10^{-8}-10^{-5} \mathrm{mmol} / \mathrm{L}\right)$ of PDGF-BB with or without PK11195/Ro5-4864 for 24 h. PK11195 and Ro5-4864 were dissolved in DMSO. FBS was protected from light, all manipulations were carried out under dim lighting, and the plates were protected from light by wrapping them in aluminum paper. The cell culture medium was changed every other day. The cells were split and subcultured after they reached confluence. Treated cells were washed with ice-cold PBS and harvested by trypsinization for further analysis.

\section{Cell proliferation assay}

The proliferation of A10 cells was analyzed using cell number counting and 3-(4,5-dimethylthiazol-2-yl)-diphenyltetrazolium bromide (MTT) analysis. For cell number counting, cells that received different treatments were trypsinized, suspended in DMEM, and counted under a microscope using a hemocytometer. For the MTT method, the number of A10 cells in each well was estimated by the uptake of the tetrazolium salt. MTT, which is selectively taken up by mitochondria, is converted to a dark blue product by living cells, but not by dead cells. After the induction of quiescence in plastic 96-well culture dishes at a density of $1 \times 10^{3}$ cells/well, the cells were incubated with the indicated drugs for $24 \mathrm{~h}$. Subsequently, $20 \mu \mathrm{L}$ of MTT $(2.5 \mathrm{~g} / \mathrm{L})$ was added to each well, and the incubation was continued for an additional $4 \mathrm{~h}$ at $37^{\circ} \mathrm{C}$. Thereafter, $150 \mu \mathrm{L}$ of DMSO was added to each well, and the absorbance at $490 \mathrm{~nm}$ was read on a microplate reader (model 680, Bio-Rad).

\section{Cell migration assay}

Wound-healing and Transwell assays were used to evaluate cell migration. For the wound-healing migration assay, A10 cells were seeded in a six-well plate at a density of $1 \times 10^{5}$ cells per well, grown to confluence, starved for $24 \mathrm{~h}$ and then pretreated with or without selective medium for another $24 \mathrm{~h}$. The cell monolayer was scratched with a small tip along a ruler and allowed to recover for $24 \mathrm{~h}$ in fresh starvation medium (serum-free DMEM) for $48 \mathrm{~h}$. The cells were visualized using an Olympus IX-70 inverted microscope (Olympus, Tokyo, Japan). The migration area (\%) was analyzed in ten randomly chosen fields under an inverted microscope by using $\mathrm{NIH}$ ImageJ software; the area at $0 \mathrm{~h} /$ the area at $48 \mathrm{~h} \times 100 \%$ was calculated. For the transwell assay, 24well tissue culture plates (BD Bioscience, Becton, NJ, USA) with an 8 - $\mu \mathrm{m}$-pore polycarbonate membrane were used. The number of migrating cells was counted in ten randomly chosen fields of duplicate chambers at a magnification of $200 \times$ for each sample.

\section{TSPO siRNA transfection}

A small interference RNA (siRNA, $50 \mathrm{nM}$ ) targeting TSPO and a scramble siRNA were transfected into A10 cells with Lipofectamine 2000 reagent (Thermo Fisher Scientific) according to the routine 
process. The assessment of silencing efficiency was performed by PCR to determine the level of TSPO RNA in A10 cells collected $48 \mathrm{~h}$ after the transfection.

Overexpression of TSPO in A10 cells

A GFP-tagged TSPO plasmid encoding the TSPO protein was constructed by Sangon Biotech Co., Ltd (Shanghai, China). The plasmid $(1 \mu \mathrm{g} / \mathrm{mL})$ was transfected into A10 cells via Lipofectamine 2000 reagent $(1 \mu \mathrm{g} / \mathrm{mL})$ in reduced serum medium for $6 \mathrm{~h}$, and then the medium was changed to DMEM containing $10 \%$ FBS to remove the Lipofectamine. After $48 \mathrm{~h}$, GFP-tagged TSPO in the A10 cells was detected by fluorescence microscopy and Western blotting.

\section{Western blotting}

Crude extracts from the dissected regions of the rat carotid arteries were washed twice with ice-cold PBS. A10 cells were treated with different substances at the indicated concentrations and times; the cells were washed once in PBS. The tissues or cell samples were lysed in RIPA buffer with a protease inhibitor mixture. After boiling the homogenates in sample buffer $(35 \mathrm{mmol} / \mathrm{L}$ Tris- $\mathrm{HCl}, \mathrm{pH} 6.8,4 \%$ SDS, $9.3 \%$ dithiothreitol, $0.01 \%$ bromophenol blue, and $30 \%$ glycerol) at $95^{\circ} \mathrm{C}$ for $5 \mathrm{~min}, 50 \mu \mathrm{g}$ of each protein sample was separated by SDS-PAGE (10\% polyacrylamide) and then electroblotted onto nitrocellulose membranes (Bio-Rad). The blots were blocked overnight with $5 \%$ nonfat dry milk in Tris-PBS with Tween 20 (TBST; $0.05 \%$ Tween 20 in $10 \mathrm{mmol} / \mathrm{L}$ of phosphate-buffered [isotonic saline]) at $4{ }^{\circ} \mathrm{C}$ with constant shaking. The blots were subsequently incubated with antibodies against TSPO (1:500), PCNA (1:800), a-SMA (1:800), p-AMPK (1:800), AMP-activated protein kinase (AMPK) (1:800), and GAPDH (1:800) overnight in a cold-room at $4{ }^{\circ} \mathrm{C}$. The membranes were then further incubated with infrared-labeled donkey anti-rabbit IRDye 800 (1:15000) at room temperature for $1 \mathrm{~h}$. The membranes were washed three times with TBST. The bound complexes were detected using the Odyssey Infrared Imaging System. The images were analyzed using the Odyssey Application Software to obtain the integrated intensities.

\section{Reverse transcriptase-PCR}

Total RNA was isolated using Trizol reagent (Invitrogen, Carlsbad, CA, USA). Total RNA $(2 \mu \mathrm{g})$ was used to synthesize CDNA, which served as a template for the amplification of TSPO and GAPDH (as a housekeeping gene). The primer sequences for TSPO were $5^{\prime}$ CCCGCTTGCTGTACCCTTACC-3' (forward) and 5'-CACCGCATACATAGTAGTTGAGCACGGTG-3' (reverse). The primer sequences for GAPDH were 5'-GTGGAGTCTACTGGCGTCTT-3' (forward) and 5'GCCTGCTTCACCACCTTCTT-3' (reverse). The amplification was performed with the following conditions: $94^{\circ} \mathrm{C}$ for $2 \mathrm{~min}$, followed by 35 cycles of denaturation at $94^{\circ} \mathrm{C}$ for $30 \mathrm{~s}$, annealing at $58^{\circ} \mathrm{C}$ for $30 \mathrm{~s}$, and extension at $72^{\circ} \mathrm{C}$ for $45 \mathrm{~s}$. This was followed by a final extension at $72{ }^{\circ} \mathrm{C}$ for $10 \mathrm{~min}$. The PCR products were electrophoresed on $2 \%$ agarose gels [18].

Statistical analysis

The data are expressed as the mean \pm SEM. Comparisons within groups were performed with one-way repeated measures ANOVA (or paired $t$-test when only two groups were compared), and comparisons among groups (or $t$-test when only two groups were compared) were performed with one-way factorial ANOVA with Holm-Sidak test. A value of $P<0.05$ was considered significant.

\section{RESULTS}

Increased TSPO expression in proliferative VSMCs in vivo and in vitro

To investigate whether TSPO is involved in neointima formation, we first measured TSPO expression in VSMCs, the most prominent cells in the formation and development of intimal hyperplasia. As shown in Fig. 1a, under physiological conditions, TSPO immunofluorescence was specifically found to overlap with VSMCs in the carotid artery. To determine the alteration of TSPO after arterial injury, we established a rat model of left carotid balloon injury. Immunofluorescence staining, immunoblotting and PCR showed that TSPO was gradually increased after balloon injury and was accompanied by the progressive thickening of the intima (Fig. 1a-c). Meanwhile, the phenotype of VSMCs switched from a contractile one to a synthetic one after injury, as evidenced by the decreased levels of a-SMA (Fig. 1a). PDGF plays an important role in the migration of VSMCs into the neointima following acute injury and in atherosclerosis [19]. In this study, PDGF-BB was employed to stimulate $A 10$ cells to mimic vascular injury in vitro. Immunoblotting analysis showed that TSPO expression was upregulated by PDGF-BB in both a concentration- and timedependent manner (Fig. 2a, b). Both PKC and PKA have been reported to regulate TSPO expression, and the release of intracellular $\mathrm{Ca}^{2+}$ stores can result in elevated levels of cAMP and PKA, which activate TSPO [20, 21]. Based on the abovementioned evidence, inhibitors of PKA, PKC, MAPK, and $\mathrm{Ca}^{2+}$ were applied to explore the underlying pathway that regulates TSPO expression. In the presence of a PKC inhibitor or a MAPK inhibitor (MAPKI), the stimulatory effect of PDGF-BB on TSPO expression was abolished (Fig. 2c, d); nevertheless, this effect was not suppressed by a PKA inhibitor or a $\mathrm{Ca}^{2+}$ inhibitor. Furthermore, PMA $\left(10^{3} \mathrm{nM}\right)$, an activator of PKC, mimicked the effect of PDGF$\mathrm{BB}$ on TSPO expression in A10 cells (Fig. 2e), suggesting that TSPO synthesis via the PKC/MAPK signaling pathway was enhanced in response to PDGF-BB stimulation in VSMCs.

Roles of TSPO and its ligands in VSMC proliferation and migration Given the evidence indicating that VSMCs play a pivotal role in neointima formation and that TSPO is robustly expressed and upregulated in VSMCs in response to vascular injury, transfection experiments were subsequently performed in vitro. A10 cells were transfected with a plasmid encoding GFP-TSPO. At $48 \mathrm{~h}$ after transfection, a higher expression levels of the TSPO protein was observed in the mitochondria of A10 cells compared with that in the control group, as determined by immunoblotting and immunofluorescence (Fig. 3a, b). The cell counting and MTT assays revealed that TSPO overexpression resulted in a significantly higher rate of proliferation in A10 cells (Fig. 3c, d). The overexpression of TSPO promoted the migration of A10 cells, as determined by the transwell and wound-healing assays (Fig. $3 e, f$ ). Furthermore, PDGF-BB treatment promoted the proliferation and migration of A10 cells that overexpressed TSPO (Supplemental Fig. 1). Loss-of-function experiments were conducted by using TSPO siRNA in A10 cells. The knockdown of TSPO was confirmed by PCR (Fig. 4a). PDGF-BB-mediated proliferation and migration were significantly reduced by TSPO knockdown in A10 cells (Fig. $4 b-d$ ). These results suggest that TSPO is at least in part responsible for PDGF-BB-mediated proliferation and migration in VSMCs.

To further confirm the role of TSPO in the aberrant growth of VSMCs, PK11195, and Ro5-4864, the two best-known TSPOspecific antagonists, were applied in vitro. As expected, treatment with PK11195 or Ro5-4864 (10,102,103,104 nM) inhibited the PDGF-BB-induced $(10 \mathrm{ng} / \mathrm{mL})$ proliferation of A10 cells in a concentration-dependent manner (Fig. 5a, b). The cell cycle, a common convergent point for mitogenic signaling cascades, is controlled by cyclins [22]. Treatment with PK11195 decreased the PDGF-BB-induced expression of cyclin D1 and cyclin E. The inhibitory effect of PK11195 on VSMC growth was further supported by parallel changes in the expression of proliferating cell nuclear antigen (PCNA, a marker for cell proliferation) after PDGF-BB stimulation (Fig. 5c, d). Moreover, treatment with PK11195 had similar effects on PDGF-BB-induced migration in A10 cells (Fig. 6a-d). 
a
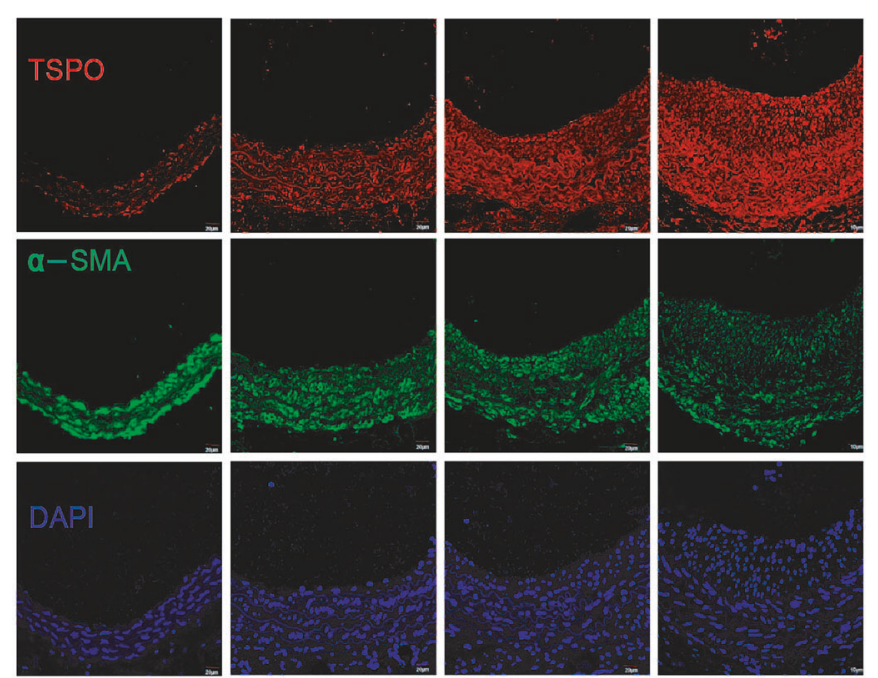

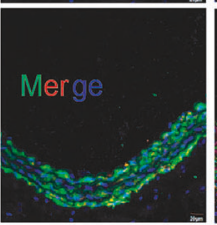

Sham

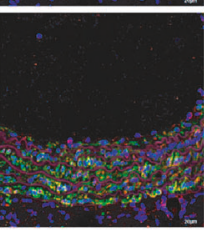

$7 d$

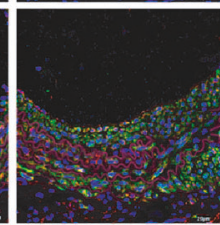

$14 \mathrm{~d}$

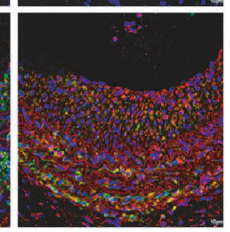

$21 d$

Injury

b

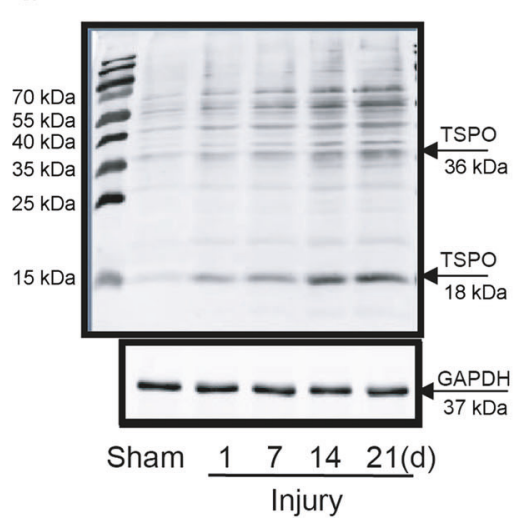

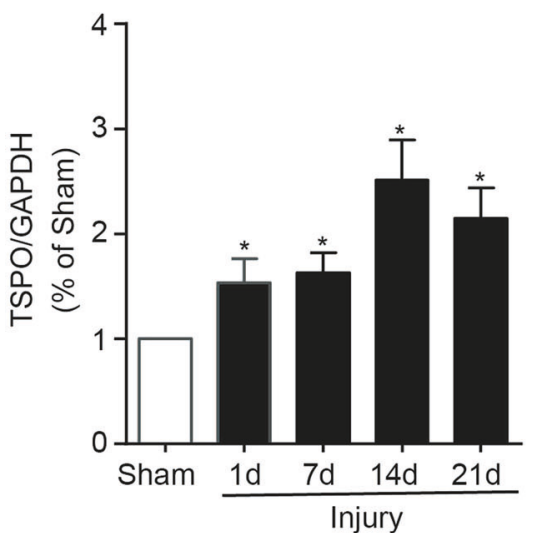

C

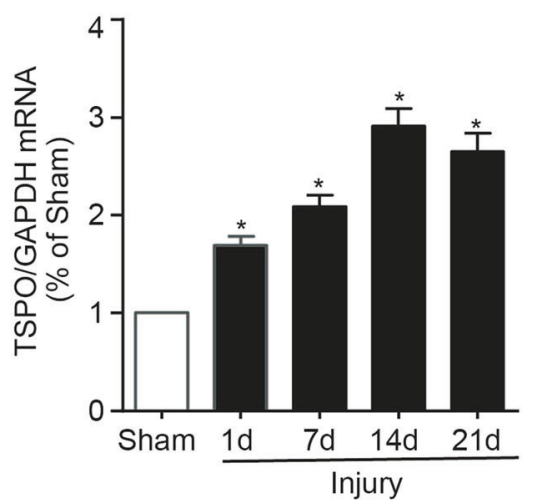

Fig. 1 Balloon injury induces neointima formation and upregulates TSPO expression in rat carotid arteries. Serial sections were obtained from carotid arteries $0,7,14$, and 21 days after balloon injury in rats. a Immunofluorescence staining for TSPO (red) and $\alpha$-SMA (green) in rat carotid arteries. The results shown are from a single experiment and are representative of 10 separate experiments. The relative fluorescence intensities of TSPO and $\alpha$-SMA are shown. $\mathbf{b}$ TSPO protein expression in rat carotid arteries was analyzed by Western blotting (WB) $(n=8)$. $\mathbf{c}$ TSPO mRNA was detected by PCR ( $n=6 ;{ }^{*} P<0.05$ vs. sham groups)

Taken together, TSPO activation is essential for the PDGF-BBinduced proliferation and migration of VSMCs. These findings indicate that TSPO ligands have potential applications in neointima formation induced by vascular injury.

Role of AMPK in the effect of a TSPO ligand on VSMCS

The evident antiproliferative effect of TSPO inhibition prompted us to investigate its underlying mechanism. TSPO, which is mainly expressed in the outer mitochondrial membrane, is involved in the modulation of energy metabolism through the regulation of ATP synthase. AMPK, which is characterized as an energy sensor (sensitive to the AMP/ATP ratio) in the regulation of glucose uptake and fatty acid oxidation throughout the body $[23,24]$, is a major upstream modulator of cell cycle progression and regulates cell proliferation in response to vascular injury $[25,26]$. The activation of AMPK requires the phosphorylation of the Thr-172 residue of this protein, which was measured in A10 cells after PK11195 treatment. Indeed, PK11195 treatment increased AMPK activity in a concentration- and time-dependent manner (Fig. 7a, b). Compound C, an AMPK-specific inhibitor, reversed the AMPK phosphorylation induced by PK11195 treatment (Fig. 7c). Neither TSPO siRNA nor GFP-TSPO affected the level of total AMPK, and the p-AMPK level was enhanced by TSPO siRNA and was suppressed when TSPO was overexpressed (Supplemental Fig. 2). Furthermore, the inhibitory effect of PK11195 on the PDGF-BBinduced proliferation and migration of $\mathrm{A} 10$ cells was blocked by 
a
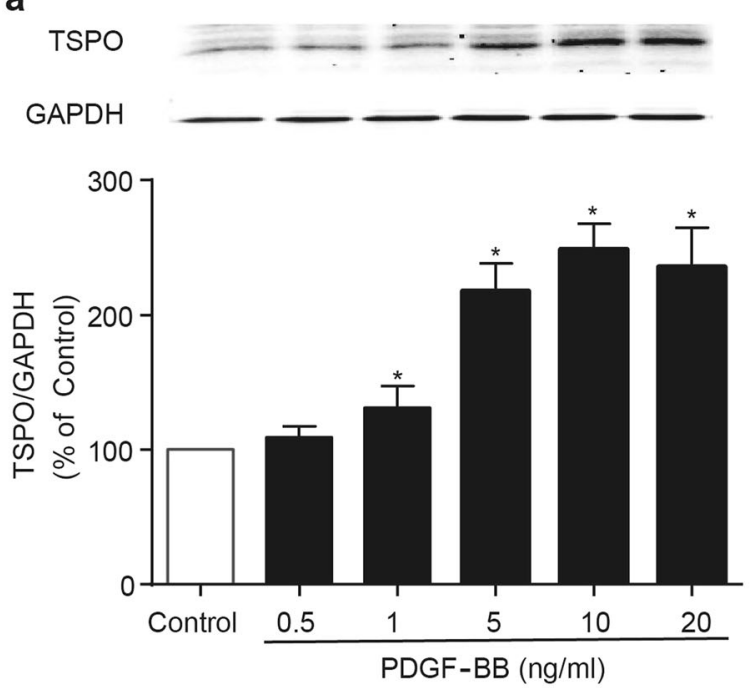

C

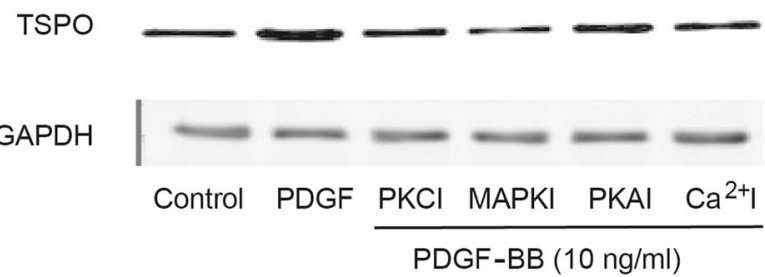

d

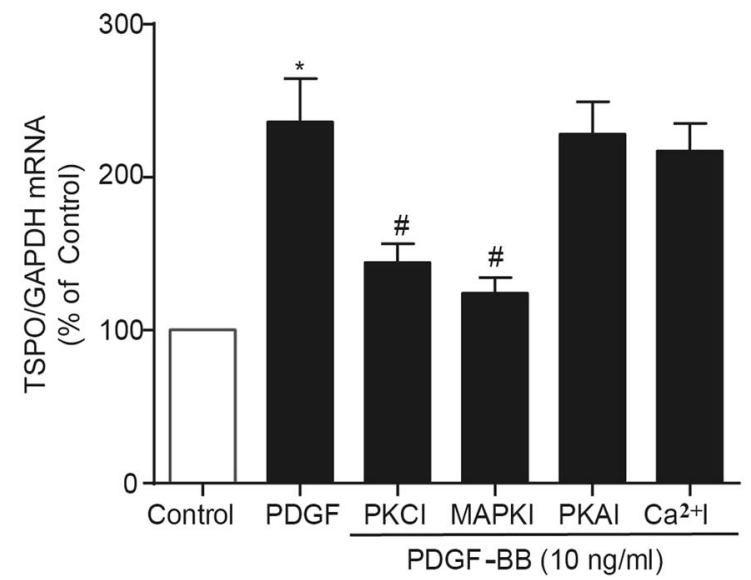

b $\mathrm{TSPO}=-\div-\div$ $\mathrm{GAPDH}-\longrightarrow-\square$
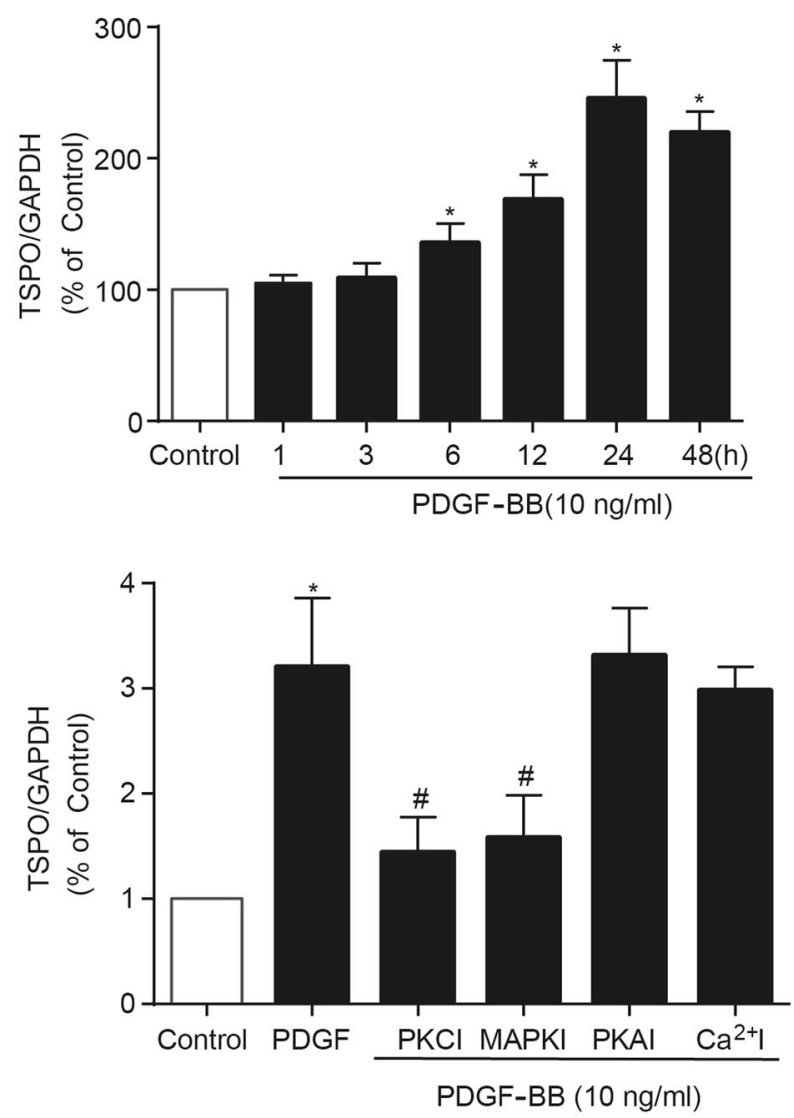

e TSPO
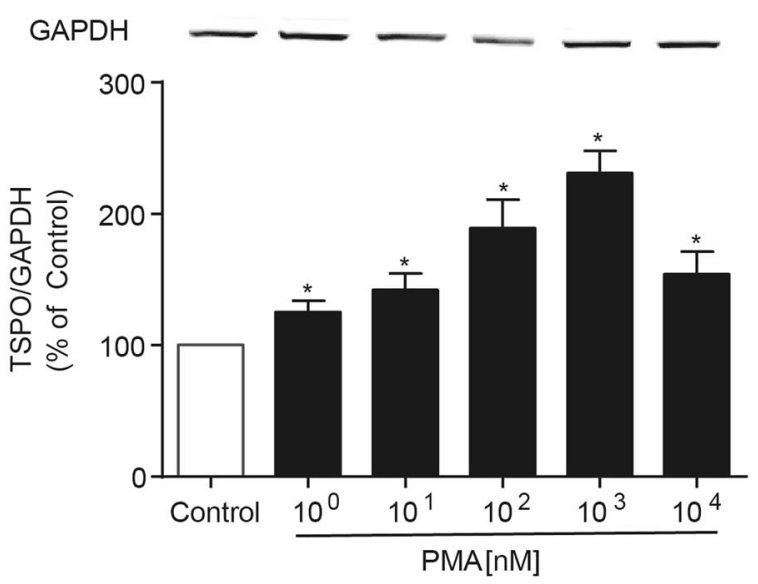

Fig. 2 Effect of PDGF-BB on the expression of TSPO protein and mRNA in VSMCs. a The effect of PDGF-BB on TSPO expression in A10 cells. A10 cells were incubated with the indicated concentrations of PDGF-BB for $24 \mathrm{~h}$. TSPO expression was determined by WB $(n=6)$. $\mathbf{b}$ The effect of PDGF-BB on TSPO expression in A10 cells. A10 cells were incubated with PDGF-BB $(10 \mathrm{ng} / \mathrm{mL})$ for the indicated times $(n=6)$. c-e The role of PKC/MAPK signaling in PDGF-BB-induced TSPO expression in A10 cells. $\mathbf{c}$, $\mathbf{d}$ The expression levels of TSPO protein and mRNA were assessed by WB and PCR after cotreatment with PDGF-BB $(10 \mathrm{ng} / \mathrm{mL})$ and PKCI $\left(10^{3} \mathrm{nM}\right)$, MAPKI $\left(10^{2} \mathrm{nM}\right), \mathrm{PKAI}\left(10^{2} \mathrm{nM}\right)$ or Ca ${ }^{2+} \mathrm{I}\left(10^{3} \mathrm{nM}\right)$ for $24 \mathrm{~h}(n=6$; ${ }^{*} P<0.05$ vs. control group; ${ }^{\#} P<0.05$ vs. PDGF-BB alone). e A10 cells were treated with the indicated concentrations of PMA $\left(1,10,10^{2}, 10^{3}\right.$, $\left.10^{4} \mathrm{nM}\right)$, a PKC agonist, for $24 \mathrm{~h}$. TSPO expression was assessed by WB. The results are expressed as the percent change compared with the control $(n=8 ; * P<0.05$ vs. control group) 
a

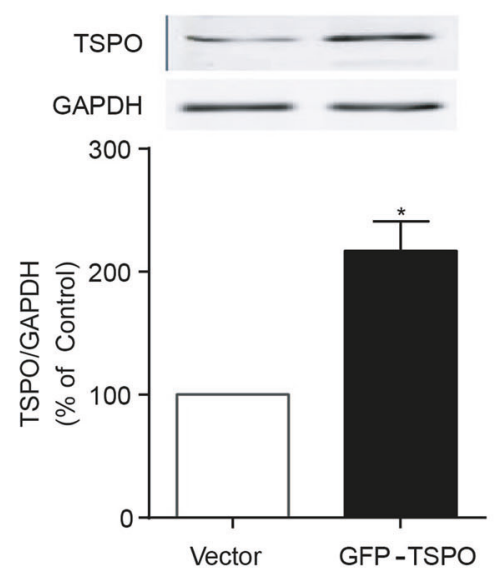

C

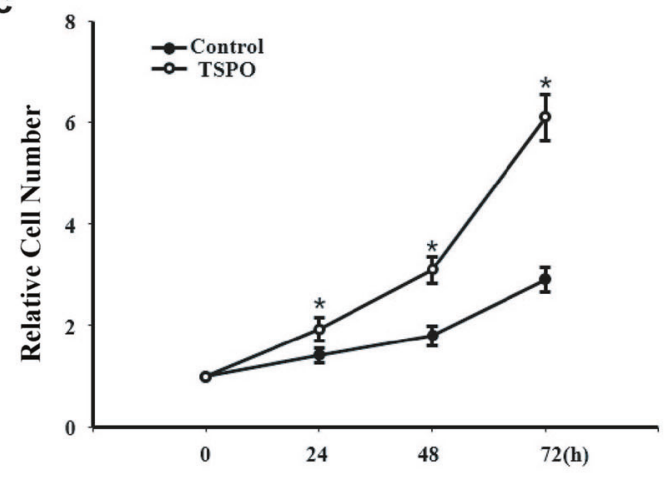

e

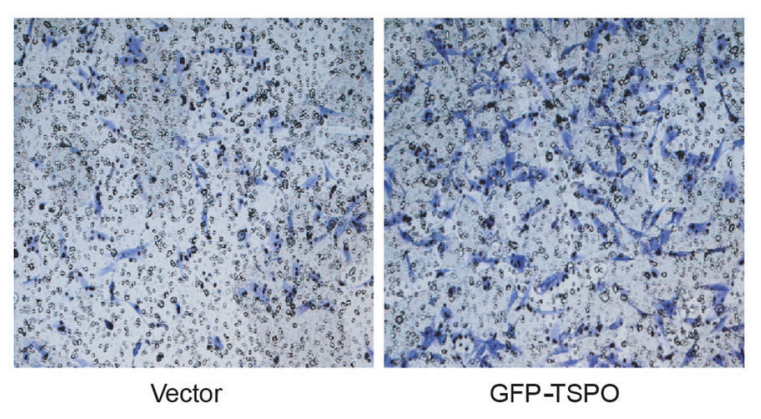

f

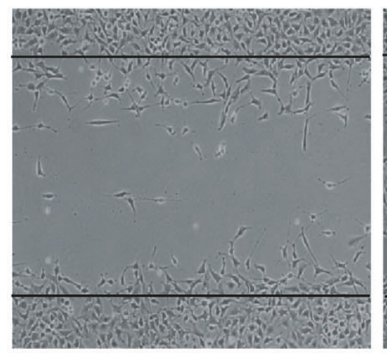

Vector

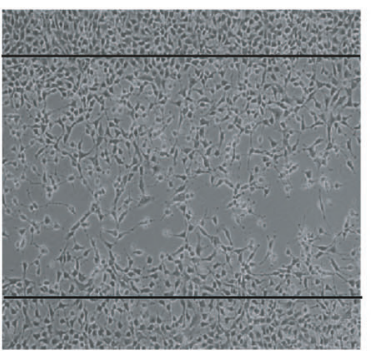

GFP -TSPO

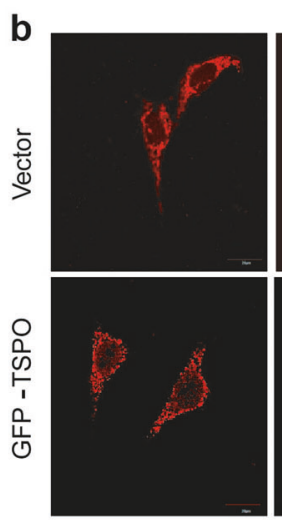

Mito-Tracker

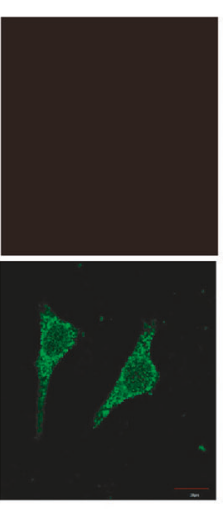

GFP-TSPO

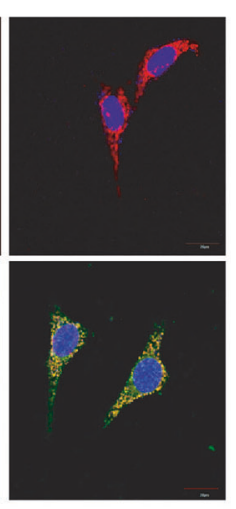

Merge

d
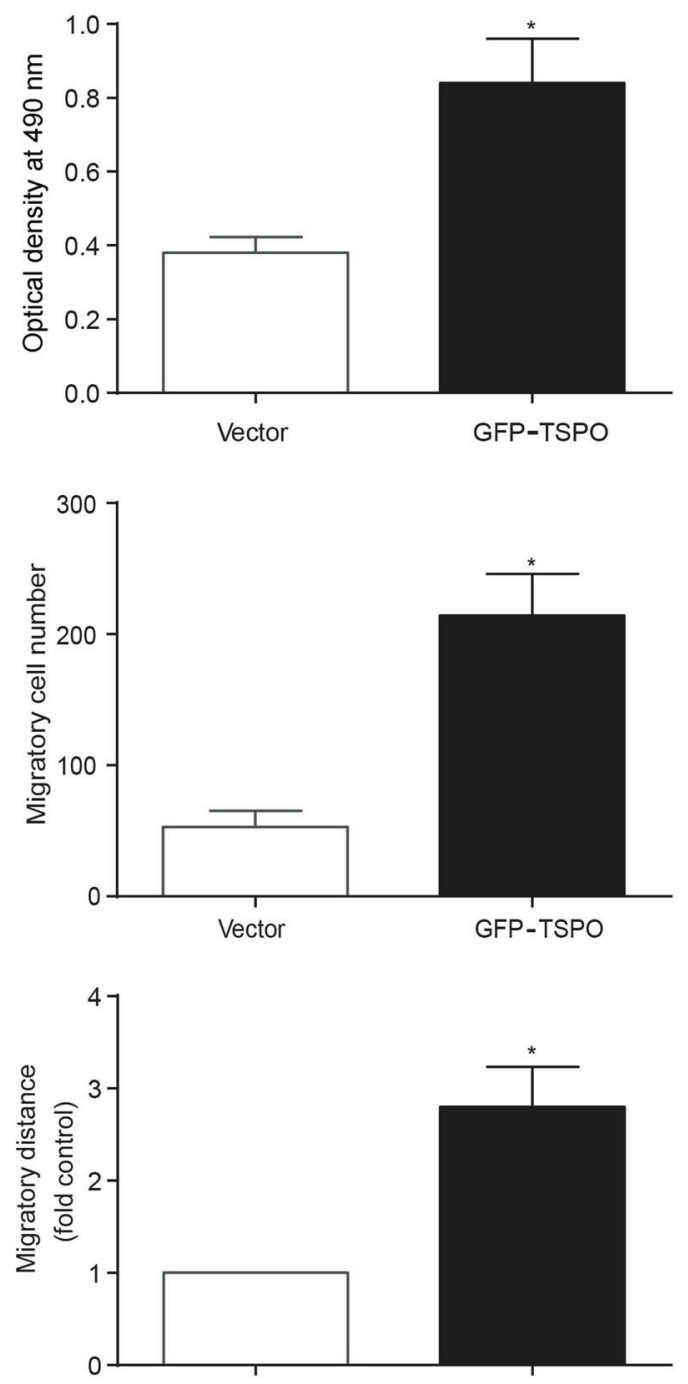

Vector

Fig. 3 Effect of the overexpression of TSPO on the proliferation and migration of VSMCs. a The overexpression of TSPO by plasmid transfection. A10 cells were transfected with GFP-tagged TSPO plasmid encoding TSPO protein or an empty vector plasmid. At $48 \mathrm{~h}$ after transfection, the cells were harvested and subjected to further assays. TSPO expression was determined by WB ( $n=6)$. b The cellular localization of overexpressed TSPO. Immunofluorescence staining revealed the colocation of TSPO (green) and Mito-Tracker (red) in A10 cells $(n=6)$. c, d The effect of the overexpression of TSPO on the proliferation of A10 cells. c Cell counting was performed $0,24,48$, and $72 \mathrm{~h}$ after TSPO plasmid transfection $(n=5)$. $\mathbf{d}$ The MTT assay was performed $48 \mathrm{~h}$ after transfection $(n=6)$. e, $\mathbf{f}$ The effect of the overexpression of TSPO on the migration of $\mathrm{A} 10$ cells $48 \mathrm{~h}$ after transfection. e, f Cell migration was assessed by the transwell and wound-healing assays. Representative images and statistical graphs are presented ( $n=10 ;{ }^{*} P<0.05$ vs. vector groups) 
40

compound $\mathrm{C}$, as determined by the MTT assay, the PCNA level and the transwell assay (Fig. 7d-f). These data indicate that the AMPK activation signaling pathway is involved in the inhibitory effect of PK11195 on VSMC growth induced by PDGF-BB.

TSPO regulator attenuates neointimal hyperplasia after balloon injury in SD rats

The finding that a TSPO-specific ligand inhibits aberrant growth in vitro prompted us to investigate its therapeutic application in vascular injury-induced restenosis. A carotid artery balloon injury model was established in SD rats. The intraperitoneal injection of PK11195 (3 mg/kg every 3 days) was started upon balloon injury in the rat carotid artery and continued for 2 weeks. HE staining

a

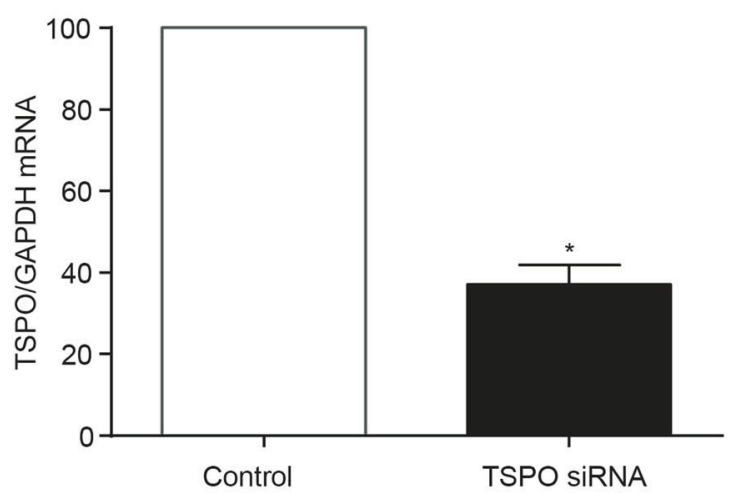

C

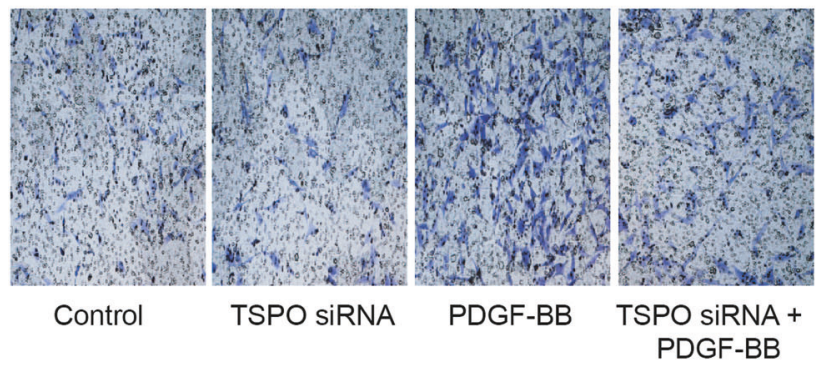

d

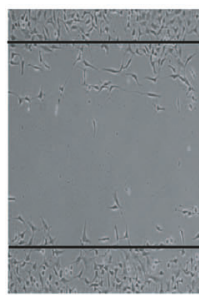

Control

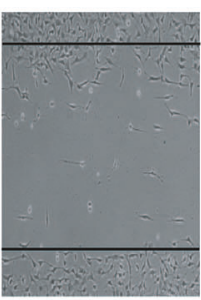

TSPO SIRNA

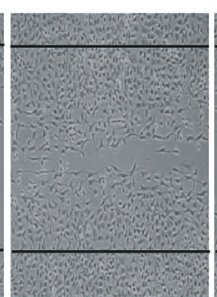

PDGF-BB

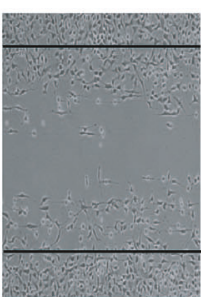

TSPO SIRNA + PDGF-BB showed evidence of the luminal narrowing of the carotid arteries 14 days after balloon injury (Fig. 8a). The neointimal area and intima-to-media (I/M) area ratio of the injured arteries were reduced in the PK11195 treatment group (Fig. 8b). Moreover, PK11195 reduced cell proliferation in the arterial sections, as evaluated by immunofluorescence of the PCNA protein (Fig. 8c). Meanwhile, PK11195 reversed the vascular injury-induced phenotype switching of VSMCs in the injured carotid arteries, as evidenced by increased a-SMA expression (Fig. 8d). These results demonstrate that this TSPO regulator is an effective pharmacological intervention to protect against balloon injury-induced neointima formation in rat carotid arteries.
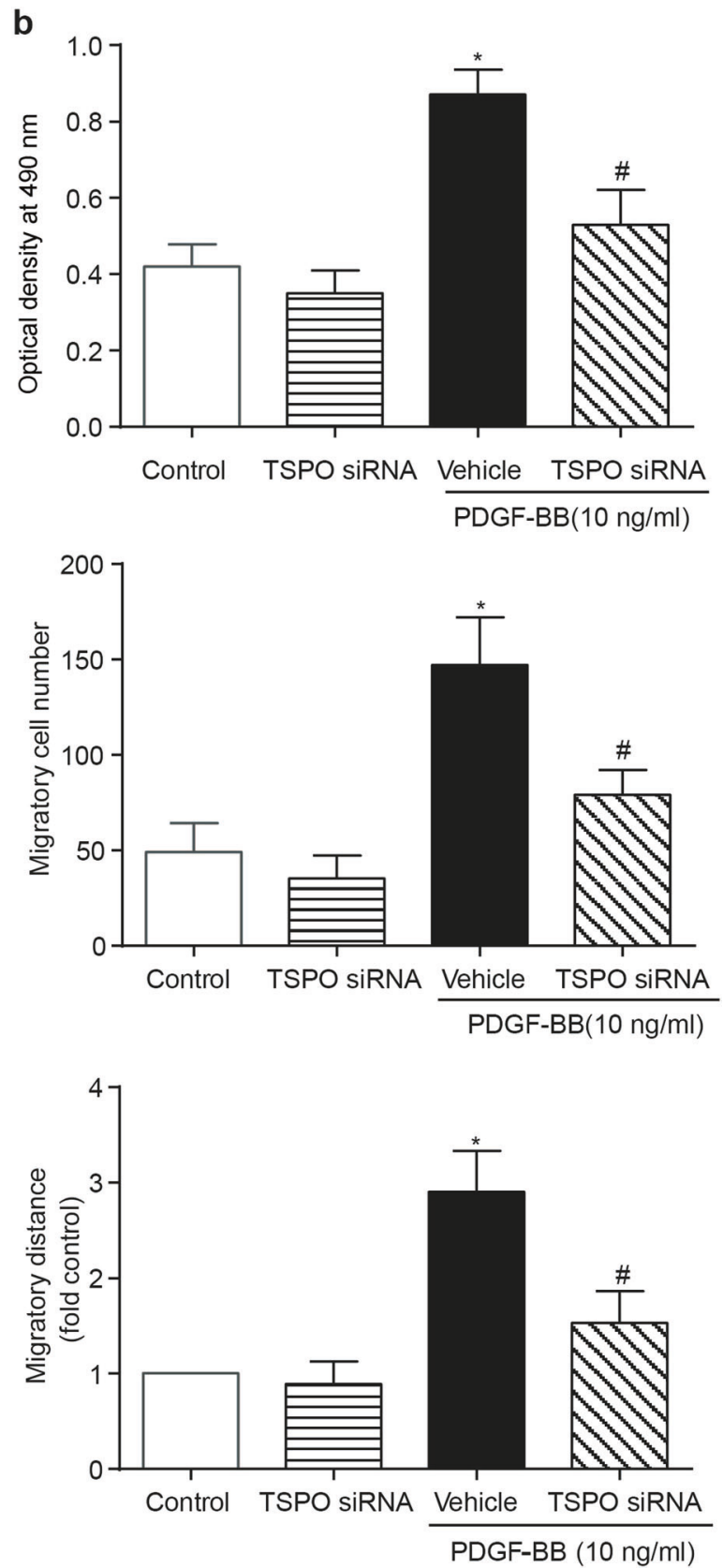

Fig. 4 Effect of TSPO knockdown on the proliferation and migration of VSMCs. A10 cells were transfected with TSPO siRNA or scramble siRNA (vehicle) for $48 \mathrm{~h}$ and then exposed to $10 \mathrm{ng} / \mathrm{mL}$ PDGF-BB for another $24 \mathrm{~h}$. The cells were harvested for various assays. a The mRNA level of TSPO was assessed by PCR $(n=6)$. b Cell proliferation was determined by the MTT assay. $\mathbf{c}$, $\mathbf{d}$ Cell migration was determined by the transwell and wound-healing assays. Representative images and statistical graphs are presented $\left(n=10 ;{ }^{*} P<0.05\right.$ vs. control, ${ }^{\#} P<0.05$ vs. vehicle transfection + PDGF-BB) 


\section{DISCUSSION}

In this study, we demonstrated for the first time that TSPO plays a crucial role in the arterial remodeling response following balloon injury. A significant increase in TSPO was observed 7 days after injury, and TSPO expression was further elevated after 21 days, whereas TSPO protein expression was limited in normal arteries. Since the intimal muscle layer of injured arteries exhibits a high rate of mitosis, the expression of TSPO may be consistent with the state of proliferation and migration of VSMCs. The increased TSPO expression was localized to the neointima of the injured arteries and was accompanied by VSMC phenotype switching, as determined by the localization of decreased a-SMA. Our results are consistent with previous reports showing an increased uptake of $\left[{ }^{11} \mathrm{C}\right] \mathrm{PK} 11195$, a TSPO radioligand, in atherosclerotic plaques $[16,27]$. The mechanism responsible for the induction of TSPO after arterial injury is unclear. Many mitogens and inflammatory cytokines have been shown to stimulate TSPO gene expression [28]. Our data showed that the growth factor PDGF-BB promoted TSPO synthesis in VSMCs, which was effectively blocked by PKC and MAPKI. Intriguingly, the PKC activator PMA mimicked the promoting effects of PDGF-BB on TSPO expression in VSMCs, further implying the pivotal role of the PKC/MAPK pathway in TSPO upregulation induced by PDGF-BB.

To determine the role of TSPO in VSMC biology, specific and effective transfection experiments were performed in vitro. After the transfection of the TSPO plasmid, increased TSPO protein was a

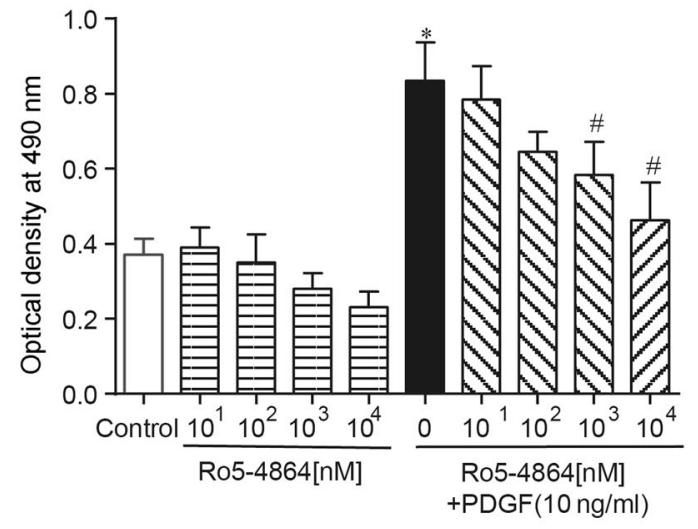

C
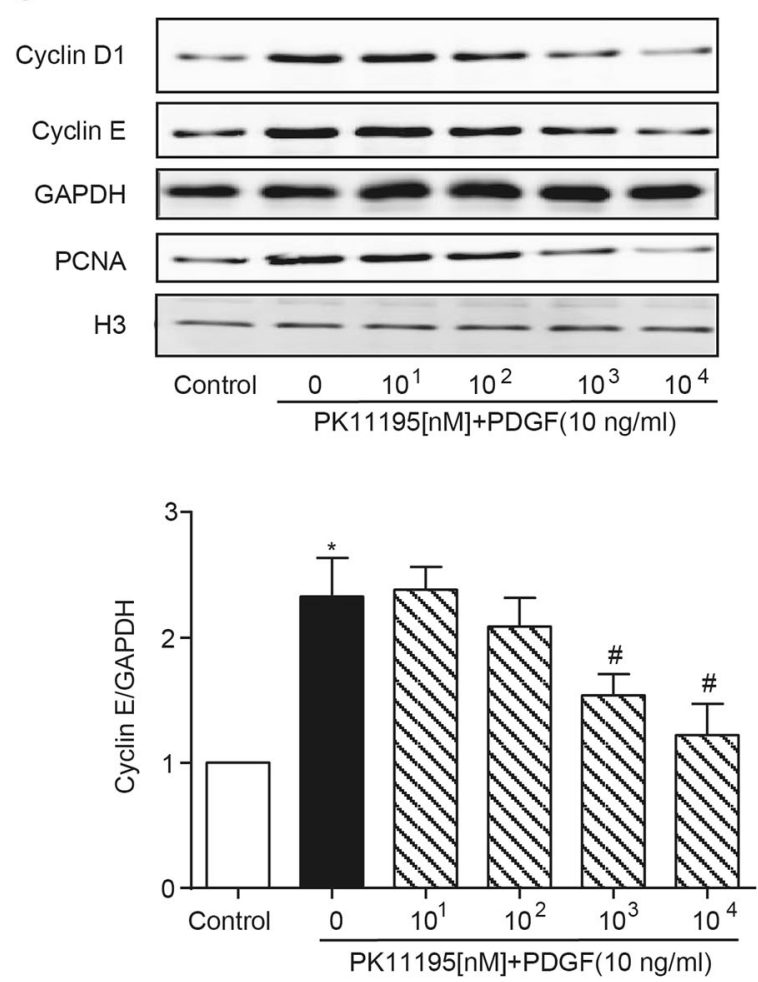

b

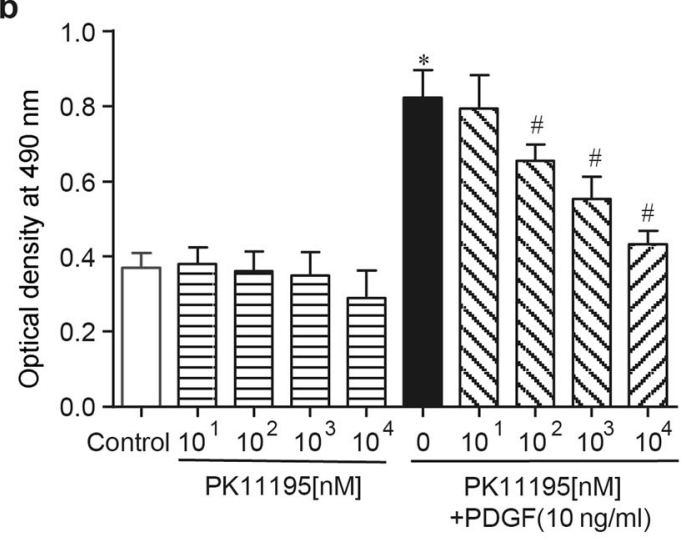

d
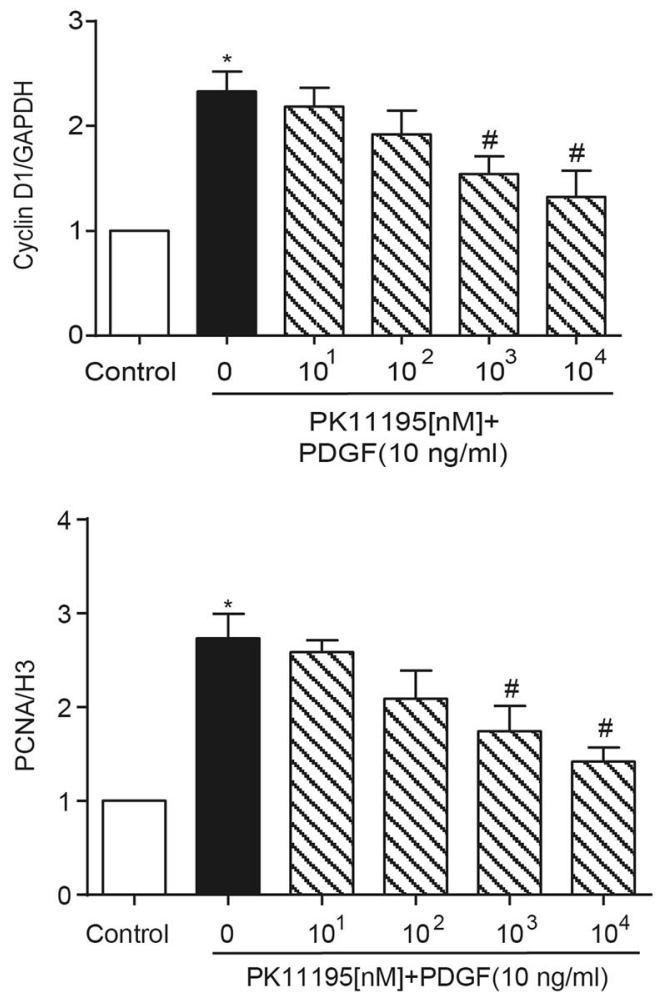

Fig. 5 TSPO ligands regulate early cell cycle progression and inhibit proliferation in PDGF-BB-treated VSMCs. A10 cells were starved and treated with various concentrations $\left(10,10^{2}, 10^{3}, 10^{4} \mathrm{nM}\right)$ of PK11195 or Ro5-4864 in the presence or absence of $10 \mathrm{ng} / \mathrm{mL}$ PDGF-BB for $24 \mathrm{~h}$. The cells were harvested and subjected to various assays. a, b The effects of Ro5-4864 and PK11195 on PDGF-BB-induced cell proliferation. Cell proliferation was determined by the MTT assay $(n=10)$. c, $\mathbf{d}$ The effect of PK11195 on cell cycle progression in PDGF-BB-treated A10 cells. A10 cells were coincubated with PK1 $1195\left(10^{4} \mathrm{nM}\right)$ and PDGF-BB $(10 \mathrm{ng} / \mathrm{mL})$ for $24 \mathrm{~h}$. The expression levels of cyclin D1, cyclin E, and PCNA were determined by WB. Histone $3(\mathrm{H} 3)$ was used as an internal control for PCNA, and GAPDH was used as an internal control for cyclin D1 and cyclin $\mathrm{E}\left(n=6 ;{ }^{*} P<0.05\right.$ vs. control, ${ }^{\#} P<0.05$ vs. PDGF-BB alone) 
a

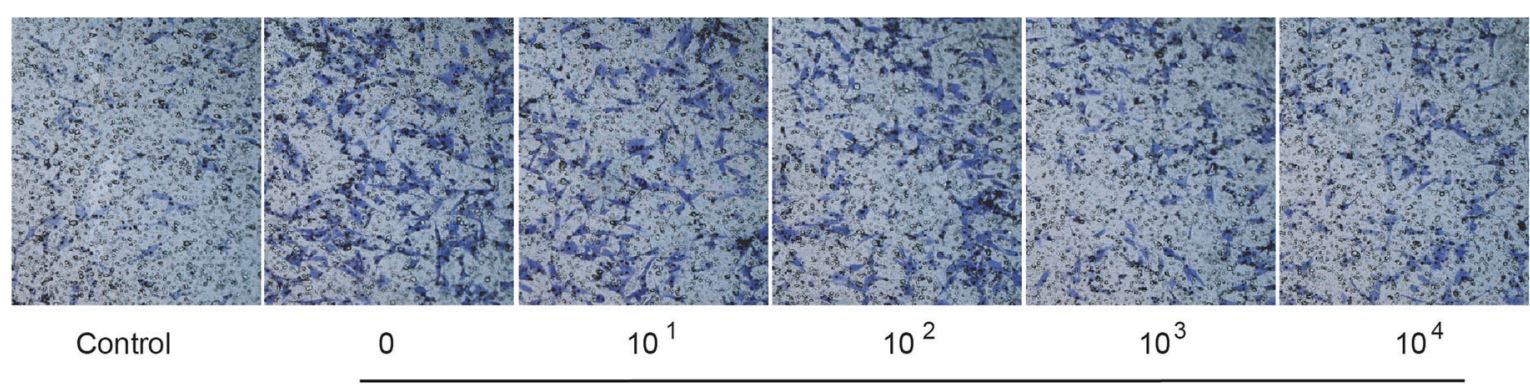

PK11195[nM]+PDGF(10 ng/ml)

b

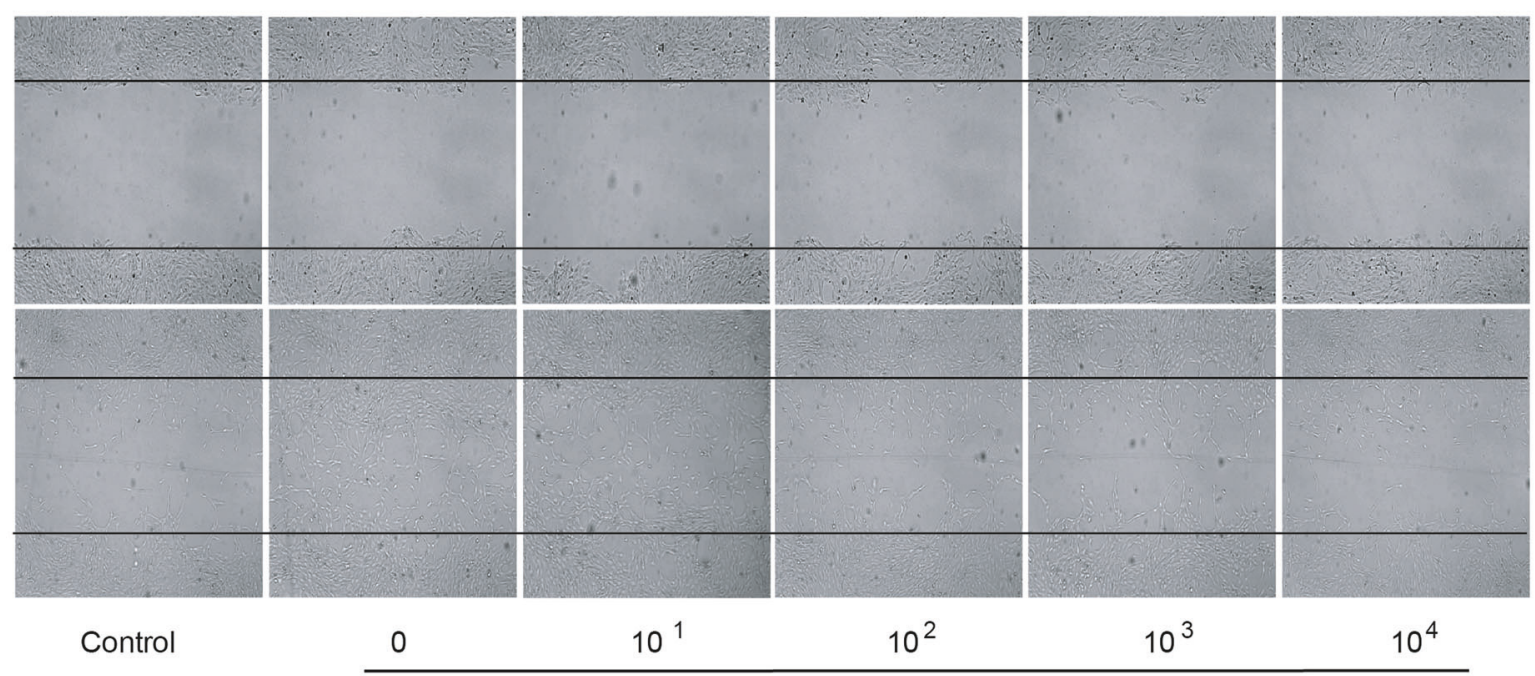

PK11195[nM]+PDGF(10 ng/ml)

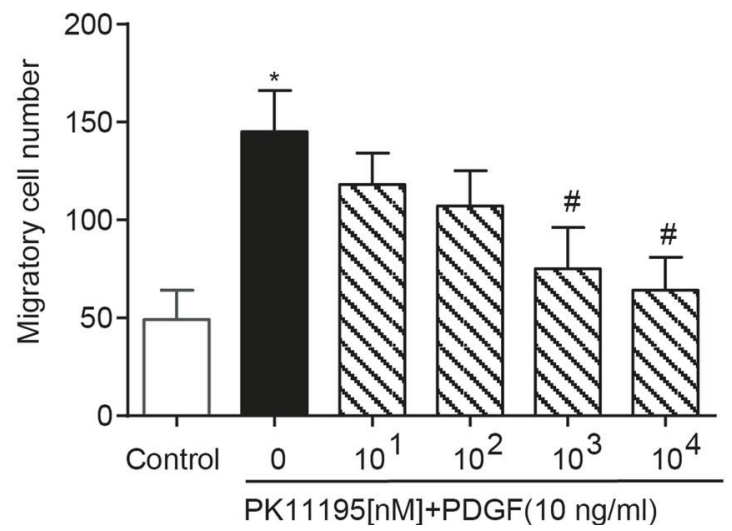

d

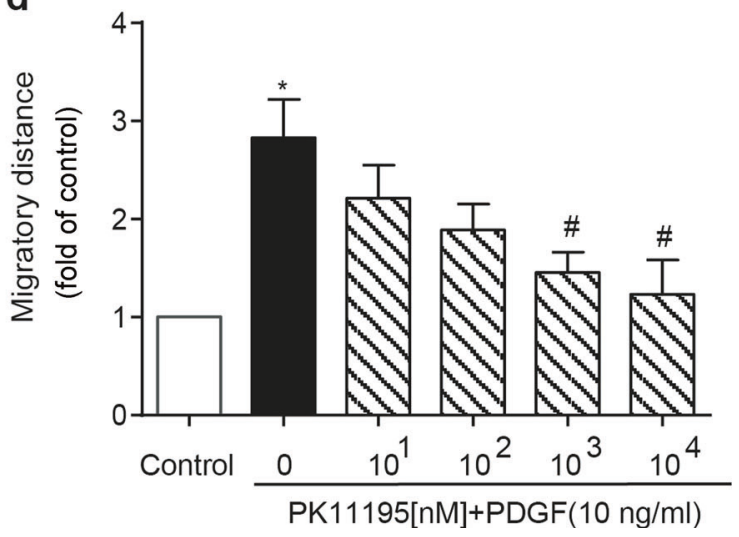

Fig. 6 Effect of PK11195 on the PDGF-BB-induced migration of VSMCs. A10 cells were starved and treated with the indicated concentrations of PK11195 in the presence or absence of $10 \mathrm{ng} / \mathrm{mL}$ PDGF-BB for $24 \mathrm{~h}$. The cells were harvested for further assays. a, b Cell migration was determined by the transwell and wound-healing assays. C, d Statistical graphs are presented $\left(n=10 ;{ }^{*} P<0.05\right.$ vs. control, ${ }^{\#} P<0.05$ vs. PDGFBB alone)

observed in the membranes of A10 cells. This finding was consistent with other reports showing that TSPO is a mitochondrial protein that mediates the transfer of cholesterol through the outer mitochondrial membrane to the inner mitochondrial membrane for steroidogenesis $[29,30]$. Moreover, the overexpression of TSPO had a positive effect on VSMC proliferation and migration. In contrast, interfering with TSPO expression with siRNA or modulating TSPO function with its ligands PK11195 and Ro5-4864 consistently resulted in a significant decrease in the proliferation and migration of PDGF-BBtreated VSMCs. Previous studies have shown that regulating the cell cycle through pharmacological intervention is considered an effective strategy for controlling VSMC proliferation [31]. Cell cycle progression is a precisely regulated process involving complex event cascades [32]. The fact that PK11195 inhibited PDGF-BB-induced VSMC proliferation suggests that several cell cycle regulatory proteins may be modulated by PK11195 treatment. As predicted, PK11195 reversed the increased expression of cyclin D1 and cyclin E in PDGF-BB-treated VSMCs, indicating that the capacity of PK11195 to induce cell cycle arrest in the early phase may be primarily responsible for its inhibition of VSMC growth. 
a

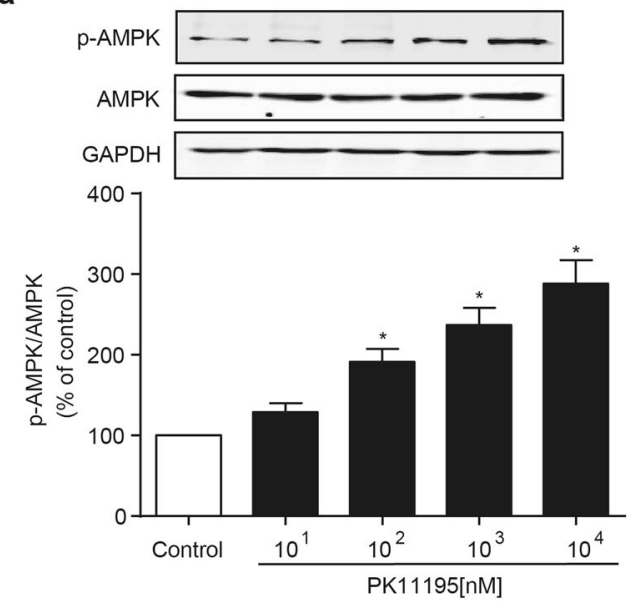

C

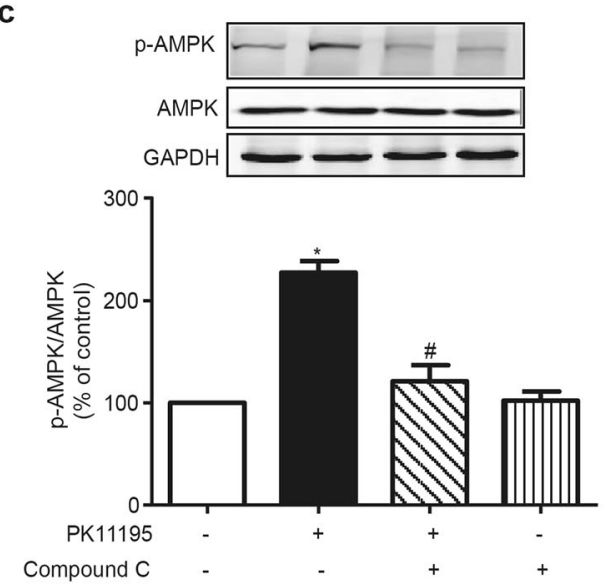

e

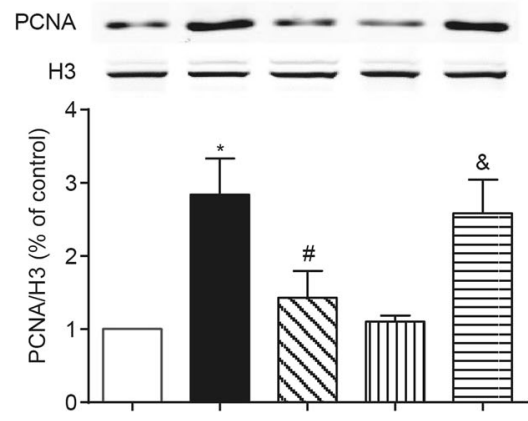

PDGF-BB

PK11195

Compound $\mathrm{C}$

Fig. 7 Role of AMPK activation in the PK11195-mediated inhibitory effect on the proliferation and migration of PDGF-BB-treated VSMCs. $\mathbf{a}$, $\mathbf{b}$ The effect of PK11195 on AMPK phosphorylation in A10 cells. A10 cells were incubated with the indicated concentrations of PK11195 for $1 \mathrm{~h}$ or with $10^{4} \mathrm{nM}$ PK11195 for the indicated time periods. The levels of p-AMPK and AMPK were determined by WB $\left(n=6 ;{ }^{*} P<0.05\right.$ vs. control). $c$ The effect of pharmacological inhibitors of AMPK on the PK11195-mediated phosphorylation of AMPK in A10 cells. A10 cells were treated with or without PK11195 $\left(10^{4} \mathrm{nM}\right)$ in the presence/absence of compound $C(10 \mu \mathrm{M})$ for $1 \mathrm{~h}$. The levels of p-AMPK and AMPK were determined by WB $\left(n=6 ;{ }^{*} P<0.05\right.$ vs. control, ${ }^{\#} P<0.05$ vs. PK11195 alone). $\mathbf{d}-\mathbf{f}$ The effect of AMPK on the PK11195-mediated inhibition of proliferation and migration in PDGF-BB-treated A10 cells. A10 cells were pretreated with or without PK11195 $\left(10^{4} \mathrm{nM}\right)$ in the presence or absence of compound $\mathrm{C}\left(10^{3} \mathrm{nM}\right)$ for $0.5 \mathrm{~h}$ and then stimulated with PDGF-BB $(10 \mathrm{ng} / \mathrm{mL})$ for $24 \mathrm{~h}$. d, e Cell proliferation was determined by the MTT assay and the PCNA level. The PCNA level was measured by WB. H3 was used as an internal control for PCNA $(n=6)$. $f$ Cell migration was measured by the transwell assay $\left(n=10 ;{ }^{*} P<0.05\right.$ vs. control, ${ }^{\#} P<0.05$ vs. PDGF-BB alone, ${ }^{8} P<0.05$ vs. PK11195 + PDGF-BB) 
AMPK has been characterized as an energy sensor in the regulation of glucose uptake and fatty acid oxidation throughout the body [23, 24]. Many studies have reported that AMPK activation has antivascular proliferation effects through inhibiting cell cycle progression $[25,26]$. Our results showed that PK11195 significantly induced AMPK activation, which was blocked by the AMPK-specific inhibitor compound C. In particular, the inhibitory effect of PK11195 on PDGF-BB-induced proliferation and migration was also prevented by compound $C$, indicating that AMPK activation mediates the pharmacological effect of PK11195 on the aberrant growth of VSMCs. AMPK is activated by reduced cellular energy status, signaled by rising AMP/ATP and ADP/ATP ratios [33]. Our findings are consistent with a recent report showing that TSPO knockout is associated with lower ATP

a

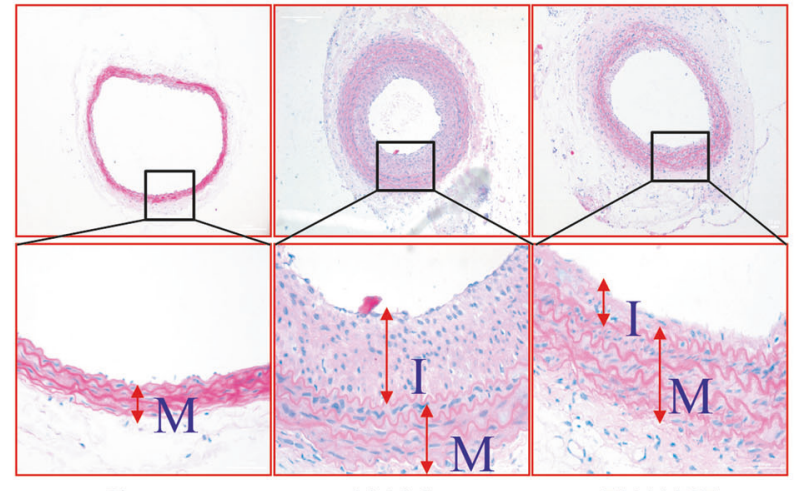

Sham

Vehicle

PK11195

C

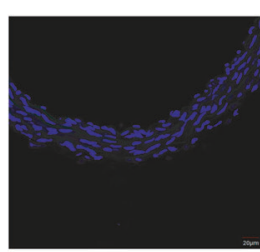

Sham

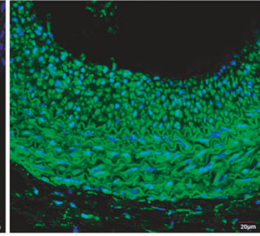

Vehicle

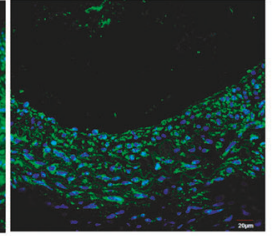

PK11195 d

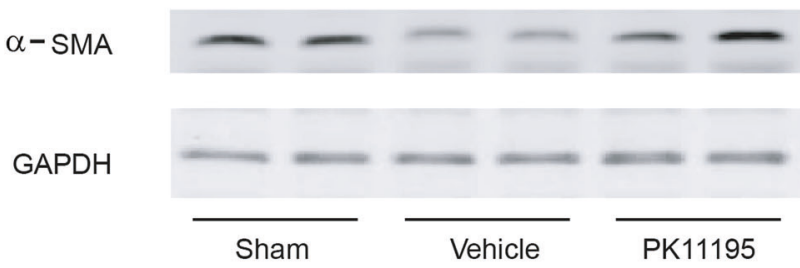

production in mouse microglia [34]. TSPO may modulate ATP synthase via a direct interaction between the ATP 'synthasome', which is composed of ATP synthase, the phosphate carrier, and ANT, and the PBR complex, which is composed of TSPO, VDAC, and ANT [34]. Notably, in the vasculature, the activation of AMPK has been shown to phosphorylate eNOS at serine-1177, stimulating NO release and the subsequent regulation of the cGMP/PKG signaling pathway [35]. Our previous observation showed that PK11195, via activating CGMP/PKG, inhibits the high glucoseinduced proliferation and migration of VSMCs. It is conceivable that AMPK is an upstream transducer in the PK11195-mediated inhibition of VSMC growth in hyperglycemia, which warrants further investigation.

b
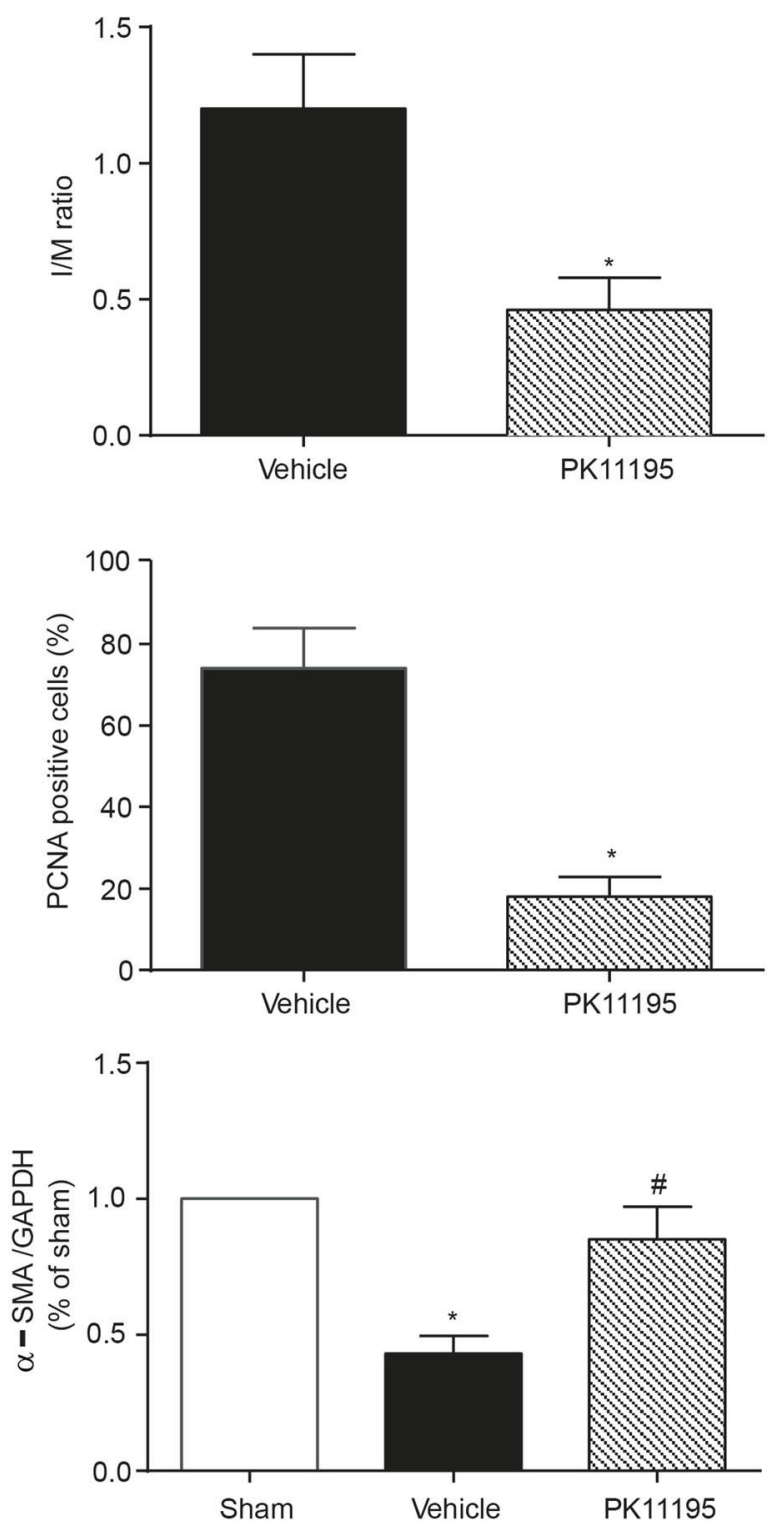

Fig. 8 Effect of PK11195 on neointima formation in balloon-injured carotid arteries in rats. Carotid arteries were collected 14 days after balloon injury from rats that received or did not receive intraperitoneal injections of PK11195 (3 mg/kg every 3 days). PK11195 administration was initiated upon the initial balloon injury. a Representative hematoxylin-eosin (HE) staining of the injured carotid arteries. b Morphometric analyses of the intima/media (I/M) area ratio. c Immunofluorescence staining of PCNA (green) and DAPI (blue) revealed the percentage of PCNA-positive cells in the neointima of the injured arteries. $\mathbf{d}$ The effect of PK11195 on the balloon injury-mediated phenotype switching of VSMCs. $\alpha$-SMA protein expression was determined by WB. Representative images and statistical graphs are shown $\left(n=6 ;{ }^{*} p<0.05\right.$ vs. sham, ${ }^{\#} P<0.05$ vs. balloon injury alone) 
The important role of TSPO in vascular remodeling during neointima formation was investigated using a rat model of balloon-induced carotid artery injury. Treatment with PK11195 for 2 weeks prevented neointimal growth in balloon-injured arteries. Neointima formation after vessel injury is a complex process involving the proliferation and migration of VSMCs [36]. In response to vascular injury, VSMCs undergo phenotypic switching, which contributes to the pathogenesis of atherosclerosis and restenosis after angioplasty [4], characterized by increased cell proliferation and the reduced expression of SMC-specific contractile proteins. In the present study, reduced PCNA expression and increased a-SMA expression were detected in the injured arteries of rats that were administered PK11195, indicating that PK11195 attenuated neointima formation by inhibiting phenotype switching and the aberrant proliferation of VSMCs induced by balloon injury. Understanding how the TSPO antagonist PK11195 acts as a potent agent to regulate VSMC cell fate in the vasculature may provide exciting new clues for its potential therapeutic application.

In summary, this study is the first to demonstrate the contribution of TSPO to the aberrant proliferation and migration of VSMCs in vivo and in vitro. Moreover, our findings show that regulating TSPO with its specific ligand can effectively prevent VSMC proliferation and neointima formation induced by balloon injury. TSPO can serve as a potential target for vascular remodeling diseases, including atherosclerosis and restenosis.

\section{ACKNOWLEDGEMENTS}

These studies were supported in part by grants from the National Key R\&D Program of China (2018YFC1312700), the National Natural Science Foundation of China (31730043, 81770425), the Program of Innovative Research Team by the National Natural Science Foundation (81721001), and the Program for Changjiang Scholars and Innovative Research Team in University; IRT1216.

\section{AUTHOR CONTRIBUTIONS}

LPW and ZFG designed the study, analyzed and interpreted the data, and drafted the paper. HW, ZSZ, MMZ, CL, HMR, and JY contributed to data acquisition. YH and CYZ designed the study and drafted the paper. All authors read and approved the final manuscript.

\section{ADDITIONAL INFORMATION}

The online version of this article (https://doi.org/10.1038/s41401-019-0293-x) contains supplementary material, which is available to authorized users.

Competing interests: The authors declare that they have no potential competing interest. This manuscript is an original contribution, and not under consideration for publication or previously published elsewhere.

\section{REFERENCES}

1. Dzau VJ, Braun-Dullaeus RC, Sedding DG. Vascular proliferation and atherosclerosis: new perspectives and therapeutic strategies. Nat Med. 2002;8:1249-56.

2. Doran AC, Meller N, McNamara CA. Role of smooth muscle cells in the initiation and early progression of atherosclerosis. Arterioscler Thromb Vasc Biol. 2008;28:812-9.

3. Li P, Zhu N, Yi B, Wang N, Chen M, You X, et al. MicroRNA-663 regulates human vascular smooth muscle cell phenotypic switch and vascular neointimal formation. Circ Res. 2013;113:1117-27.

4. Owens GK, Kumar MS, Wamhoff BR. Molecular regulation of vascular smooth muscle cell differentiation in development and disease. Physiol Rev. 2004;84:767-801.

5. Gatliff J, East DA, Singh A, Alvarez MS, Frison M, Matic I, et al. A role for TSPO in mitochondrial $\mathrm{Ca}^{2+}$ homeostasis and redox stress signaling. Cell Death Dis. 2017;8:e2896.

6. Rupprecht R, Papadopoulos V, Rammes G, Baghai TC, Fan J, Akula N, et al. Translocator protein (18 kDa) (TSPO) as a therapeutic target for neurological and psychiatric disorders. Nat Rev Drug Discov. 2010;9:971-88.
7. Mendonca-Torres MC, Roberts SS. The translocator protein (TSPO) ligand PK11195 induces apoptosis and cell cycle arrest and sensitizes to chemotherapy treatment in pre- and post-relapse neuroblastoma cell lines. Cancer Biol Ther. 2013;14:319-26.

8. Chen MK, Guilarte TR. Translocator protein $18 \mathrm{kDa}$ (TSPO): molecular sensor of brain injury and repair. Pharmacol Ther. 2008;118:1-17.

9. Zhou T, Dang Y, Zheng YH. The mitochondrial translocator protein, TSPO, inhibits HIV-1 envelope glycoprotein biosynthesis via the endoplasmic reticulumassociated protein degradation pathway. J Virol. 2014;88:3474-84.

10. Beinlich A, Strohmeier R, Kaufmann M, Kuhl H. Specific binding of benzodiazepines to human breast cancer cell lines. Life Sci. 1999;65:2099-108.

11. Repalli J. Translocator protein (TSPO) role in aging and Alzheimer's disease. Curr Aging Sci. 2014;7:168-75.

12. Gaemperli O, Shalhoub J, Owen DR, Lamare F, Johansson S, Fouladi N, et al. Imaging intraplaque inflammation in carotid atherosclerosis with ${ }^{11} \mathrm{C}-\mathrm{PK} 11195$ positron emission tomography/computed tomography. Eur Heart J. 2012;33:1902-10.

13. Tsamatsoulis M, Kapelios CJ, Katsaros L, Vakrou S, Sousonis V, Sventzouri S, et al. Cardioprotective effects of intracoronary administration of 4-chlorodiazepam in small and large animal models of ischemia-reperfusion. Int J Cardiol. 2016;224:90-5.

14. Wang HJ, Fan J, Papadopoulos V. Translocator protein (Tspo) gene promoterdriven green fluorescent protein synthesis in transgenic mice: an in vivo model to study Tspo transcription. Cell Tissue Res. 2012;350:261-75.

15. Bird JL, Izquierdo-Garcia D, Davies JR, Rudd JH, Probst KC, Figg N, et al. Evaluation of translocator protein quantification as a tool for characterising macrophage burden in human carotid atherosclerosis. Atherosclerosis. 2010;210:388-91.

16. Laitinen I, Marjamaki P, Nagren K, Laine VJ, Wilson I, Leppanen P, et al. Uptake of inflammatory cell marker [11C]PK11195 into mouse atherosclerotic plaques. Eur J Nucl Med Mol Imaging. 2009;36:73-80.

17. Htun NM, Chen YC, Lim B, Schiller T, Maghzal GJ, Huang AL, et al. Near-infrared autofluorescence induced by intraplaque hemorrhage and heme degradation as marker for high-risk atherosclerotic plaques. Nat Commun. 2017;8:75. doi: 10.1038/s41467-017-00138-x.

18. Yu C, Wang Z, Han Y, Liu Y, Wang WE, Chen $C$, et al. Dopamine $D_{4}$ receptors inhibit proliferation and migration of vascular smooth muscle cells induced by insulin via down-regulation of insulin receptor expression. Cardiovasc Diabetol. 2014;13:97. doi: 10.1186/1475-2840-13-97.

19. Raines EW. PDGF and cardiovascular disease. Cytokine Growth Factor Rev. 2004;15:237-54.

20. Batarseh A, Li J, Papadopoulos V. Protein kinase C epsilon regulation of translocator protein (18 kDa) Tspo gene expression is mediated through a MAPK pathway targeting STAT3 and c-Jun transcription factors. Biochemistry. 2010;49:4766-78.

21. Chen C, Kuo J, Wong A, Micevych P. Estradiol modulates translocator protein (TSPO) and steroid acute regulatory protein (StAR) via protein kinase A (PKA) signaling in hypothalamic astrocytes. Endocrinology. 2014;155:2976-85.

22. Sherr CJ. Cancer cell cycles. Science. 1996;274:1672-7.

23. Carling D, Viollet B. Beyond energy homeostasis. the expanding role of AMPactivated protein kinase in regulating metabolism. Cell Metab. 2015;21:799-804.

24. Qi D, Young LH. AMPK. energy sensor and survival mechanism in the ischemic heart. Trends Endocrinol Metab. 2015;26:422-9.

25. Igata M, Motoshima H, Tsuruzoe K, Kojima K, Matsumura T, Kondo T, et al. Adenosine monophosphate-activated protein kinase suppresses vascular smooth muscle cell proliferation through the inhibition of cell cycle progression. Circ Res. 2005;97:837-44.

26. Ewart MA, Kennedy S. AMPK and vasculoprotection. Pharmacol Ther. 2011; 131:242-53.

27. Hellberg S, Silvola JMU, Kiugel M, Liljenback $H$, Savisto $N$, Li XG, et al. 18-kDa translocator protein ligand ${ }^{18} \mathrm{~F}$-FEMPA: biodistribution and uptake into atherosclerotic plaques in mice. J Nucl Cardiol. 2017;24:862-71.

28. Guilarte TR. TSPO in diverse CNS pathologies and psychiatric disease: a critical review and a way forward. Pharmacol Ther. 2018;194:44-58.

29. Batoko H, Veljanovski V, Jurkiewicz P. Enigmatic translocator protein (TSPO) and cellular stress regulation. Trends Biochem Sci. 2015;40:497-503.

30. Banati RB, Middleton RJ, Chan R, Hatty CR, Kam WW, Quin C, et al. Positron emission tomography and functional characterization of a complete PBR/TSPO knockout. Nat Commun. 2014;5:5452.

31. Peng C, Zhang S, Liu H, Jiao Y, Su G, Zhu Y. A newly synthesized Ligustrazine stilbene derivative inhibits PDGF-BB induced vascular smooth muscle cell phenotypic switch and proliferation via delaying cell cycle progression. Eur J Pharmacol. 2017;814:106-13.

32. Xie N, Chen M, Dai R, Zhang Y, Zhao H, Song Z, et al. SRSF1 promotes vascular smooth muscle cell proliferation through a Delta133p53/EGR1/KLF5 pathway. Nat Commun. 2017;8:16016. 
46

33. Lin SC, Hardie DG. AMPK: sensing glucose as well as cellular energy status. Cell Metab. 2018;27:299-313.

34. Liu GJ, Middleton RJ, Kam WW, Chin DY, Hatty CR, Chan RH, et al. Functional gains in energy and cell metabolism after TSPO gene insertion. Cell Cycle. 2017; 16:436-47.
35. Sun W, Lee TS, Zhu M, Gu C, Wang Y, Zhu Y, et al. Statins activate AMP-activated protein kinase in vitro and in vivo. Circulation. 2006;114:2655-62.

36. Malik N, Francis SE, Holt CM, Gunn J, Thomas GL, Shepherd L, et al. Apoptosis and cell proliferation after porcine coronary angioplasty. Circulation. 1998; 98:1657-65. 